\title{
Closure Theorem for Partially Semialgebraic Sets
}

by

\author{
Stanisław ŁOJASIEWICZ and María-Angeles ZURRO
}

Presented by Józef SICIAK

Summary. In 1988 it was proved by the first author that the closure of a partially semialgebraic set is partially semialgebraic. The essential tool used in that proof was the regular separation property. Here we give another proof without using this tool, based on the semianalytic $L$-cone theorem (Theorem 2), a semianalytic analog of the CartanRemmert-Stein lemma with parameters.

The authors began work on this joint paper in September 2002, but in November 2002 a heart attack put an end to Stanisław Lojasiewicz's life. The second author wishes to honour Professor Stanisław Eojasiewicz with this paper.

1. Let $M$ be an analytic manifold. If $X$ is a finite-dimensional real vector space of dimension $n$, then an analytic function $f: M \times X \rightarrow \mathbb{R}$ is said to be an $X$-polynomial if for an open neighborhood $U$ of any point of $M$, in some (and then any) linear coordinate system $X \rightarrow \mathbb{R}_{t}^{n}$ the restriction $f_{U \times X}$ is a polynomial in $t$ with coefficients analytic on $U$.

A subset $E \subset M \times X$ is said to be $X$-semialgebraic if every point $a \in M$ has an open neighborhood $U$ such that $E_{U}$ is described in $U \times X$ by $X$ polynomials. This definition implies that finite unions and finite intersections of $X$-semialgebraic sets are $X$-semialgebraic.

When $Y$ is another finite-dimensional real vector space, an analytic mapping $M \times X \rightarrow Y$ is said to be an $X$-polynomial mapping if in some (and then any) linear coordinate system in $Y$ its components are $X$-polynomials.

2000 Mathematics Subject Classification: Primary 32B20; Secondary 14P15, 32C25.

Key words and phrases: semianalytic sets, closure theorem.

Work supported by the European Community's Human Potential Programme under contract HPRN-CT-2001-00271, RAAG. 
If $N$ is a real analytic manifold, $Y$ a finite-dimensional real vector space, $f: M \rightarrow N$ an analytic mapping and $g: M \times X \rightarrow Y$ an $X$-polynomial mapping, then the inverse image under the mapping $(x, v) \mapsto(f(x), g(x, v))$ of a $Y$-algebraic subset of $N \times Y$ is an $X$-semialgebraic subset of $M \times X$.

2. Let $X$ be a topological space and let $\mathcal{A}$ be an algebra of continuous real functions on it. We say that a subset $E$ of $X$ is described by $\mathcal{A}$ if

$$
E=\bigcup_{i=1}^{s} \bigcap_{j=1}^{r} E_{i j}
$$

where $E_{i j}$ is of the form $\left\{f_{i j}>0\right\}$ or $\left\{f_{i j}<0\right\}$ or $\left\{f_{i j}=0\right\}$ with $f_{i j} \in \mathcal{A}$.

Let $S(\mathcal{A})$ be the class of subsets of $X$ which are described by $\mathcal{A}$. Note that $\mathcal{A}[t]$ is an algebra of continuous real functions on $X \times \mathbb{R}$. Let $\pi: X \times \mathbb{R} \rightarrow X$ be the natural projection.

The following facts have been proved in [2, pp. 105-110].

Proposition 1. The connected components theorem for $S(\mathcal{A})$ implies:

(1) The connected components theorem for $S(\mathcal{A}[t])$.

(2) $E \in S(\mathcal{A}[t]) \Rightarrow \pi(E) \in S(\mathcal{A})$.

Let $\mathcal{A}_{U}$ be the ring of analytic functions on a relatively compact neighborhood $U$ of a given point $a \in M$ which have analytic extensions onto neighborhoods of $\bar{U}$. Then, by the connected components theorem, each set in $S\left(\mathcal{A}_{U}\right)$ has a finite number of connected components. Hence, by induction, we obtain the following statement:

(1) Each set in $S\left(\mathcal{A}_{U}\left[t_{1}, \ldots, t_{m}\right]\right)$ has a finite number of components.

(2) If $E \in S\left(\mathcal{A}_{U}\left[t_{1}, \ldots, t_{m}\right]\right)$, then $\pi(E)$ is semianalytic in $M$, where $\pi: M \times \mathbb{R}^{k} \rightarrow M$ is the natural projection for $k=1, \ldots, m$.

As consequences, using linear coordinate systems, we obtain the following connected components theorem and generalized Tarski-Seidenberg theorem.

Let $X$ be a finite-dimensional real vector space. We say a family $\mathcal{F}$ of subsets of $M \times X$ is locally $X$-finite if every point of $M$ has a neighborhood $U$ such that the family of non-empty traces of the sets of $\mathcal{F}$ on $U \times X$ is finite. Observe that the union of any locally $X$-finite family of $X$-semialgebraic subsets of $M \times X$ is $X$-semialgebraic.

Connected Components Theorem. All connected components of an $X$-semialgebraic set $E \subset M \times X$ are $X$-semialgebraic and their family is locally $X$-finite. Any open-closed subset of $E$ is $X$-semialgebraic.

Now, let $Y$ be a finite-dimensional real vector space and let $\pi: M \times X \times Y$ $\rightarrow M \times X$ be the natural projection. 
Tarski-Seidenberg Theorem. If $E \subset M \times X \times Y$ is $(X \times Y)$ semialgebraic, then $\pi(E)$ is $X$-semialgebraic (in $M \times X)$.

3. Let $M$ be a real analytic manifold and let $L$ be a finite-dimensional real vector space. We say that a semianalytic set $E \subset M \times L$ is an $L$-cone if each fiber $E_{x}$ is a cone, for $x \in M$. This is equivalent to saying that $c_{\lambda}(E)=E$ for $\lambda>0$, where $c_{\lambda}=\operatorname{id}_{M} \times \lambda \operatorname{id}_{L}: M \times L \rightarrow M \times L$.

We have the following straightforward properties:

(1) The closure of an $L$-cone is an $L$-cone.

(2) Finite unions, intersections and differences of $L$-cones are $L$-cones.

(3) The subset of points of dimension $k$ of a semianalytic $L$-cone is a semianalytic $L$-cone.

(4) The set of smooth points of dimension $k$ of an $L$-cone is a semianalytic $L$-cone.

We define an $L$-conical germ to be any set germ $A$ at $(a, 0) \in M \times L$ satisfying $c_{\lambda}(A)=A$ for any $\lambda \in \mathbb{R}, \lambda>0$. Clearly, the germ at $(a, 0)$, with $a \in M$, of any $L$-cone $C$ in $M \times L$ is $L$-conical, and we have $\operatorname{dim}_{(a, 0)} C=$ $\operatorname{dim} C_{(a, 0)}$. Observe that for $L$-cones $C, D$, we have

$$
C \subset D \Leftrightarrow C_{(a, 0)} \subset D_{(a, 0)} \text { for all } a \in M
$$

and also

$$
C=D \Leftrightarrow C_{(a, 0)}=D_{(a, 0)} \text { for all } a \in M .
$$

Lemma 1. For any L-conical semianalytic germ $A$ at $(a, 0) \in M \times L$ there is a semianalytic $L$-cone $C$ in $U \times L$, where $U$ is an open neighborhood of a such that $C_{(a, 0)}=A$. If $A$ is analytic, so is $C$.

Proof. Let $E$ be a semianalytic representative of $A$ in a neighborhood $U \times \Omega$ of $(a, 0)$. For any $u \in U$ and $v \in L$ with $|v|=1$ there is a maximal segment $(0, r(u, v)) v$ which is contained in $E_{u}$ or disjoint from $E_{u}\left({ }^{1}\right)$. As $A$ is $L$-conical, we must have $r=\inf \{r(u, v): u \in W,|v|=1\}>0$, with a relatively compact semianalytic neighborhood $W \subset U$ of $a$. Then, if $0<s<r$, the set

$$
D=E \cap(W \times\{|x|<s\}) \subset M \times L
$$

is a union of some segments $u \times(0, s) v$ or $u \times[0, s) v$, with $|v|=1$. It follows that $C=\bigcup_{\lambda>0} c_{\lambda}(D)$ is a semianalytic $L$-cone $\left({ }^{2}\right)$ such that $C_{(a, 0)}=A$.

We will need the following fact:

Proposition 2. Any decreasing sequence $E_{\nu}$ of analytic subsets of $M$ is locally stationary, i.e. each point $a \in M$ has a neighborhood $U$ such that the sequence $E_{\nu} \cap U$ is stationary.

$\left({ }^{1}\right)$ Since $E_{u} \cap \mathbb{R} v$ is semianalytic.

$\left({ }^{2}\right)$ Its germ at any point is the germ of some $c_{\lambda}(D)$ (at this point). 
Proof. Since at any point $c \in M$ the ring $\mathcal{A}_{c}$ of analytic germs at $c$ is noetherian, the sequence of germs $\left(E_{\nu}\right)_{0}$ is stationary, i.e. for some $p$ we have $\left(E_{\nu}\right)_{0}=\left(E_{p}\right)_{0}$ for all $\nu \geq p$. Taking a stratification at $c$ compatible with $E_{p}\left({ }^{3}\right)$, i.e. such that $E_{p} \cap U$ is the union of some of its leaves, and using a sort of the identity principle: if an open non-empty subset of a connected analytic leaf $\Lambda$ is contained in $E_{p}$, then $\Lambda \subset E_{p}$, we get the result.

We have the following real analog of the Cartan-Remmert-Stein lemma with parameters.

We say that a subset $E$ of $M \times L$ is L-algebraic if each $a \in M$ has an open neighborhood $U$ such that $E$ is defined (by equalities) by polynomials in $x \in L$ with coefficients analytic in $U$ (it can always be defined by one function).

THEOREM 1. Each analytic L-cone in $M \times L$ is L-algebraic.

Proof. Fix any $a \in M$. Our $L$-cone $E$ is defined in some open neighborhood $W=U \times V$ of $(a, 0)$ by an analytic function $f=\sum_{i=0}^{\infty} h_{i}$, where $h_{i}$ is a form of degree $i$ with coefficients analytic in $U$. For any $(x, v) \in U \times V$ we have the equivalence

$$
f(x, v)=0 \Leftrightarrow h_{i}(x, v)=0, i=0,1,2, \ldots
$$

In fact, if $f(x, v)=0$, then, as $E$ is an $L$-cone, we have $0=f(x, t v)=$ $\sum_{i=0}^{\infty} h_{i}(x, v) t^{i}$ for $t>0$ small enough, hence $h_{i}(x, v)=0, i=0,1, \ldots$ The sequence of analytic sets $E_{k}=\left\{(x, v) \in U \times V: h_{i}(x, v)=0, i=\right.$ $1, \ldots, k\}$ is decreasing, hence by Proposition 2 , it must be locally stationary, so, diminishing $W$, we have $\{f=0\} \cap W=E_{k}$ for some $k$. Thus the sets $E$ and $E_{k}$ are equal in $W$, and so, being $L$-cones, they must be equal in $U \times L$.

Proposition 3. For any semianalytic germ $S$ at a point $a \in M$, there exists the smallest analytic germ at a which contains $S$. Moreover, it has the same dimension as $S$.

Proof. Let $\mathcal{A}$ be the ring of germs of analytic functions at $a$ and $\mathcal{I}$ the ideal of germs which vanish on $S$. As $\mathcal{A}$ is noetherian, the ideal $\mathcal{I}$ is generated by germs at $a$ of some analytic functions $f_{1}, \ldots, f_{s}$ in a neighborhood of $a$. Then the analytic germ

$$
\left\{f_{1}=\cdots=f_{s}=0\right\}_{a}
$$

is as required. The second part follows by taking a normal stratification compatible with a representative of $S$.

Proposition 4. Any semianalytic L-cone is contained in an algebraic L-cone of the same dimension.

$\left({ }^{3}\right)$ E.g. a normal one (see [4]). 
Proof. Let $E$ be our semianalytic $L$-cone. Let $a \in M$. By Proposition 3 we take the smallest analytic germ $S$ which contains $E_{(a, 0)}$. It is $L$-conical since also $c_{\lambda}(S)$ is such a germ (in view of $\left.c_{\lambda}\left(E_{(a, 0)}\right)=E_{(a, 0)}\right)$. By Lemma 1 there is an analytic $L$-cone $C$ such that $C_{(a, 0)}=S$. It has the same dimension as $E$ and it is algebraic, by Theorem 1 .

4. We will now need some other facts from semianalytic geometry. For any subset $E$ of a topological space $X$, we define a decreasing sequence of closed sets $V_{i}=V_{i}(E), i=1,2, \ldots$, as follows. We define by recursion $E_{0}=E$ and $E_{i+1}=\bar{E}_{i} \backslash E_{i}$, and we put $V_{i}=V_{i}(E)=\bar{E}_{i}$. In particular, $V_{0}=\bar{E}$ and $V_{0} \supset V_{1} \supset \cdots$.

Lemma 2. If $V_{2 r}=\emptyset$, then $E=\left(V_{0} \backslash V_{1}\right) \cup \cdots \cup\left(V_{2 r-2} \backslash V_{2 r-1}\right)$.

Proof. In fact, $E_{i}=\bar{E}_{i} \backslash E_{i+1}$, and also

$$
\left(V_{i} \backslash V_{i+1}\right) \cup\left(V_{i+1} \backslash E_{i+1}\right)=\bar{E}_{i} \backslash \bar{E}_{i+1} .
$$

Hence, for $p$ even, we have

$$
E_{2 r-p}=\left(V_{2 r-p} \backslash V_{2 r-p+1}\right) \cup\left(V_{2 r-p+2} \backslash V_{2 r-p+3}\right) \cup \cdots \cup\left(V_{2 r-2} \backslash V_{2 r-1}\right),
$$

and we get the result for $p=2 r$.

Proposition 5. A set $E \subset M$ is semianalytic if and only if the sets $V_{i}=V_{i}(E)$ are closed semianalytic and $V_{s}=\emptyset$ for some $s$. Then $V_{i+1}$ is nowhere dense in $V_{i}, i=0,1, \ldots$, so $\operatorname{dim} V_{i} \leq \operatorname{dim} E$, and we have $V_{i}=\emptyset$ for $i>n=\operatorname{dim} M$. If $2 r>n$, then

$$
E=\left(V_{0} \backslash V_{1}\right) \cup \cdots \cup\left(V_{2 r-2} \backslash V_{2 r-1}\right) .
$$

Observe that if $E$ is a cone, so are the $V_{i}$ 's.

Proof. Indeed, by Lemma 2, the condition is sufficient. Now suppose that the set $E$ is semianalytic. Then the sets $V_{i}$ are semianalytic, $V_{i+1}$ is nowhere dense in $V_{i}, i=0,1, \ldots$, and $V_{i}=\emptyset$ for $i>n$ (see [4, II.5, II.7]). Applying Lemma 2 we get $(*)$. The last statement follows from the definition of $V(E)$.

Proposition 6. If $E$ is semianalytic in $M$, then the set

$$
\left\{x \in M: \operatorname{dim}_{x} E=k\right\}
$$

is semianalytic.

Proof. In fact, it is sufficient to take a distinguished (or normal) stratification of an interval $Q$, compatible with $E$ (see [1]). Then the points of our set in $Q$ are precisely those which belong to closures (in $Q$ ) of leaves of dimension $k$ and do not belong to the closure of any leaf of dimension $>k$.

REMARK 1. If a semianalytic set is of constant dimension $k$, then so is its closure. 
5. We have the following semianalytic analog of the Cartan-RemmertStein lemma with parameters.

THEOREM 2. Any semianalytic L-cone is L-semialgebraic.

Proof. Let $E$ be a semianalytic $L$-cone in $M \times L$. We proceed by induction on the dimension $k$ of $E$. By Proposition 5, we have

$$
E=\left(F_{0} \backslash F_{1}\right) \cup \cdots \cup\left(F_{p-1} \backslash F_{p}\right),
$$

where the $F_{i}$ are closed semianalytic $L$-cones of dimension $\leq k$. In view of Proposition 6 and Remark 1, for each $F=F_{i}$ we have

$$
F=S_{k} \cup \cdots \cup S_{0},
$$

with $S_{i}$ a closed semianalytic $L$-cone of constant dimension $i$.

By Proposition 4 , any $L$-cone $S_{i}$ is contained in an $L$-algebraic $L$-cone $C_{i}$ of dimension $i$. Let $S_{i}^{*}$ the set of non-smooth points of $S_{i}$. It is a closed semianalytic $L$-cone of dimension $<i<k$, and it is $L$-semialgebraic by the induction hypothesis. Then $S_{i} \backslash S_{i}^{*}$ is an analytic submanifold of dimension $i$; it is dense in $S_{i}$ (as the latter is of constant dimension $i$ ). Next the set $C_{i}^{*}$ of points of $C_{i}$ which are not smooth of dimension $i$ is a closed $L$-semialgebraic $L$-cone of dimension $<i$, and $C_{i} \backslash C_{i}^{*}$ is an analytic submanifold.

The set $T=S_{i}^{*} \cup C_{i}^{*}$ is closed semialgebraic of dimension $<i$. Then $S_{i} \backslash T$ is closed in $C_{i} \backslash T$, but it is also open (in the latter set) as both are analytic submanifolds of dimension $i$. Hence $S_{i} \backslash T$ is a locally $L$-finite union of connected components of $C_{i} \backslash T$, and so it is $L$-semialgebraic. Since $S_{i}$ is the closure of $S_{i} \backslash T$, it must be $L$-semialgebraic. Consequently, so are the $F_{i}$ 's and $E$.

6. Let $X, Y$ be finite-dimensional real vector spaces. Consider the hyperplane $H=X \times Y \times 1 \subset X \times Y \times \mathbb{R}$. Let $F$ be a subset of $X \times Y$, and put

$$
c(F)=\bigcup_{(x, y) \in F}\{x\} \times \mathbb{R}_{+}(y, 1),
$$

with $\mathbb{R}_{+}=(0, \infty)$. Hence, $c(F) \cap H=F \times 1$.

REMARK 2. Let $E \subset X \times Y$ be a $Y$-cone. Then

$$
\widetilde{E}=\{(x, y, z, t):(x, y) \in E, z=t y, t>0\} \subset X \times Y \times Y \times \mathbb{R}
$$

is a $(Y \times \mathbb{R})$-cone, and $c(E)=\mu(\widetilde{E})$, where $\mu(x, y, z, t)=(x, z, t)$ for $(x, y, z, t)$ in $X \times Y \times Y \times \mathbb{R}$. Thus, by the Tarski-Seidenberg theorem, if $E$ is a $Y$-semialgebraic $Y$-cone then $c(E)$ is a $Y$-semialgebraic $(Y \times \mathbb{R})$-cone.

Remark 3. Let $M=X \times Y \times \mathbb{R}_{+}$and consider $E \subset X \times Y$. Then $\operatorname{cl}_{M}(c(E))=c\left(\operatorname{cl}_{X \times Y} E\right)$.

This yields the following equivalence: 
Proposition 7. Let $E \subset X \times Y$ be any $Y$-cone. Then $E$ is $Y$-semialgebraic if and only if $c(E)$ is semianalytic.

Proof. Consider the $(Y \times \mathbb{R})$-cone $\widetilde{E}$ as in Remark 2. It is $(Y \times Y)$ semialgebraic. By the Tarski-Seidenberg theorem $\mu(\widetilde{E})$ is $Y$-semialgebraic, hence $c(E)$ is semianalytic. Conversely, since $c(E) \cap H=E \times 1$, the set $E$ is semianalytic. Hence, by Theorem 2, $E$ is $Y$-semialgebraic.

Closure Theorem. The closure of any $Y$-semialgebraic subset of $X \times Y$ is $Y$-semialgebraic.

Proof. Let $A$ be a $Y$-semialgebraic set. Put $E=c(A)$. Then $E$ is a $Y$-cone and it is $Y$-semialgebraic. By Proposition 7, the cone $c(E)$ is semianalytic. Thus $\overline{c(E)}$ is semianalytic (by the closure theorem for semianalytic sets, see [4, II.5, Cor. 5.2]). The set $\bar{E}$ is a $Y$-cone, and it is also $Y$-semialgebraic, in view of Remark 3 and Proposition 7 . Remark 3 implies that $c(\bar{A})$ is $Y$ semialgebraic. Hence so is $\bar{A}$, since $\bar{A} \equiv c(\bar{A}) \cap H$.

\section{References}

[1] K. Kurdyka, S. Łojasiewicz et M.-A. Zurro, Stratifications distinguées comme outil en géométrie semianalytique, Manuscripta Math. 113 (1997), 81-102.

[2] S. Łojasiewicz, Ensembles semi-analytiques, preprint IHES, 1965.

[3] —, Sur l'adhérence d'un ensemble partiellement semi-algébrique, Publ. Math. IHES 68 (1988), 205-210.

[4] S. Łojasiewicz y M.-A. Zurro, Una introducción a la geometría semi- y sub-analítica, Publicaciones de la Universidad de Valladolid, 1993.

María-Angeles Zurro

Departamento de Matemáticas

Universidad Autónoma de Madrid

28049 Madrid, Spain

E-mail: mangeles.zurro@uam.es 